\title{
The Key Technologies and Applications of Power Fiber to the Home in Yunnan
}

\author{
Hu Jinsong ${ }^{1, a}$ Sun Yanzhi ${ }^{1, b}$, *, Tian Zhijun ${ }^{1}$ and Liu Xuan ${ }^{1}$ \\ ${ }^{1}$ Yunnan Electric Power Dispatch and Control Center \\ ${ }^{1}$ Kunming, Yunnan, 650000, P.R. China \\ ahujinsong_yn@sina.com, bepsonsun@126.com \\ *Corresponding author
}

Keywords: PFTTH, OPLC, xPON, Smart grid, Specific system applications.

\begin{abstract}
The power market reform energetically promotes the implement of the power fiber to the home (PFTTH) systems, and provides unprecedented opportunities to it. PFTTH, based on the optical-fiber core network, is outstanding in link bandwidth, time delay and reliability. PFTTH can offer intelligent control and convenient interaction, which benefits the diversity of electric power communication services. Based on the PFTTH experimental project in Yunnan Yuxi, this paper details the network architecture and the design of the whole system scheme. And the key technologies in PFFTH are analysed, including optical fibre composite low-voltage cable (OPLC) and xPON technology. With passive optical network technology, the PFTTH experimental project provides the feasibility to develop communication services, such as measurement automation, intelligent power service, internet access and network security, which are all introduced detailly.
\end{abstract}

\section{Introduction}

The power fiber to the home (PFTTH) system combined with passive optical network technology, supports a variety of smart grid services, and promotes the informatization, automation and interaction of the smart grid [1]. Under the background of the power reform, PFTTH has became the second batch pilot project of the State Grid Corporation of China in 2010. China Southern Power Grid Corp also energetically promotes the implement of PFTTH.

By the end of 2016, the cable network of Yunnan Province has covered 3710 grid nodes, including station, business, office, switch station, power station, etc. The length of cable network cable in total in $49365 \mathrm{~km}$. However, the average utilization rate of core resources is only $36.31 \%$, thus there is a unique advantage in the development of network and communication resources on potential. Therefore, in the reform of electric power, the PFTTH experimental project in Yuxi is constructed. The project aims to promote the sharing of resources, improve the utilization rate of state-owned assets, and take full use of existing fiber infrastructures.

With passive optical network technology, the PFTTH experimental project realizes the remote information acquisition metering, home electrical analysis, intelligent control, and the application of green clean energy. Yunnan Power Grid Corp considers smart grid on the basis of triple play, promotes the integration of smart grid, telecommunication network, radio network, television 
network, and Internet. The information interactive function between the user and the community, or the 95598 system is also considered.

The structure of the paper is as follows: in Section 2, we demonstrate the overall network architecture of the PFTTH experimental project and details the design of the whole system scheme. The key technologies for PFTTH are introduced in Section 3. The realization of the system applications are described in Section 4. In Section 5, the conclusions are summarized.

\section{Network Architecture}

According to the experimental project, the PFTTH system divides the overall architecture into three layers: service layer, network layer and cable layer. The service layer provides measurement automation service, intelligent power service and Internet service. The network layer is used to solve the problem of distribution remote communication and distribution local communication PON. And the cable layer is divided into two parts: $10 \mathrm{kV}$ distribution cable and the part of PFTTH.

The overall architecture is shown in figure 1. Where, ODF is Optical Distribution Frame, MODF is Main Optical Distribution Frame.

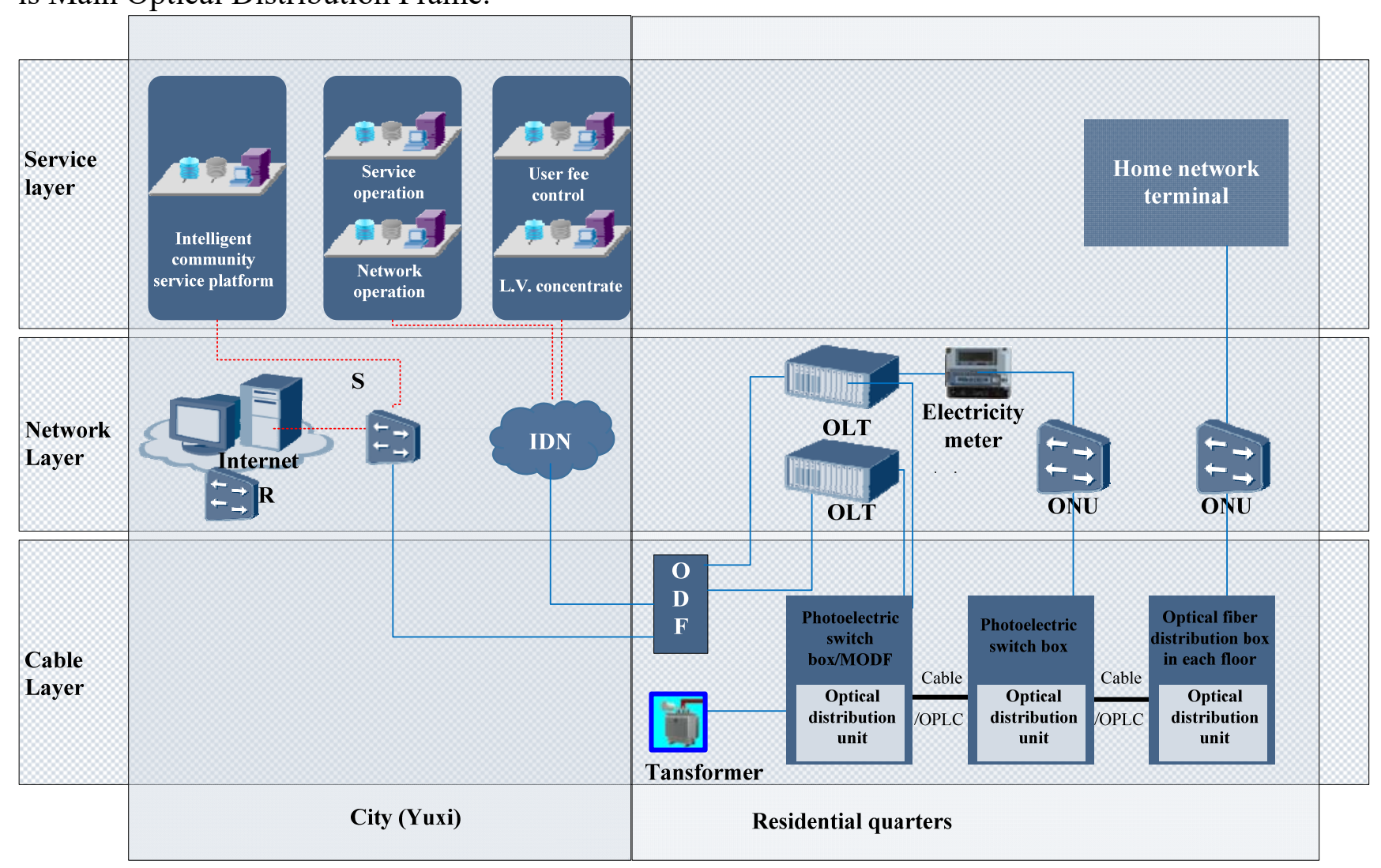

Figure 1. Overall network architecture of PFTTH

\section{Key Technologies}

PFTTH is a method to link the communication between station terminals and end users by employing the optical medium in OPLC. A PFTTH system is composed of XPON central office, optical cable distribution point, user access point and XPON terminal. OPLC lays the optical fiber and low-voltage cable to reach each electric meter at each home. Working with xPON (passive optical network) technology, PFTTH brings about the two-way interaction of electric energy data of 
smart power consumption and "triple play" services. So as to ensure power supplement as well as the transmission of the "last mile" information in power grid.

\subsection{OPLC}

OPLC integrates optical units (consisting of optical fibers and beam tubes) into low-voltage $(0.4 \mathrm{kV})$ cables [2]. It can realize both power transmission and optical communication at the same time, brings about the simultaneous transmission of information.

Typical and common OPLC structure cross section diagram is shown in figure 2.

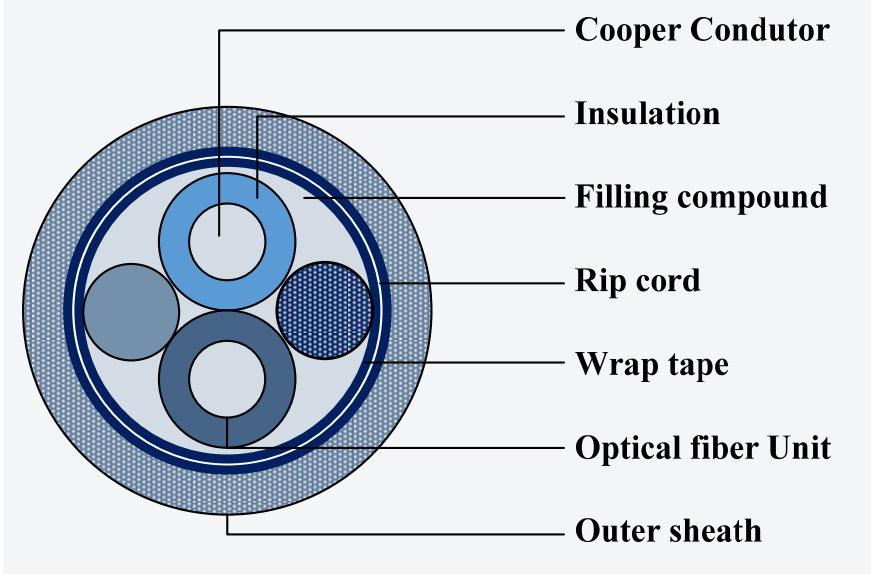

Figure 2. Typical structure of OPLC

From figure 2, the main materials include copper condutor, insulation, filling compound, rip cord, wrap tape, optical fiber unit and outer sheath. As reported, the cost of power optical fiber composite cable is less than traditional low-voltage about $10 \% \sim 20 \%$, which can reduce the total cost by $40 \%[3]$.

\subsection{Network of PFTTH}

The Internet access service uses the PON technology in the PFTTH system. PON consists of the optical line terminal OLT (Optical Line Terminal), ONU (optical network unit), and ODN (optical distribution network).

Power system PON network is shown in figure 3:

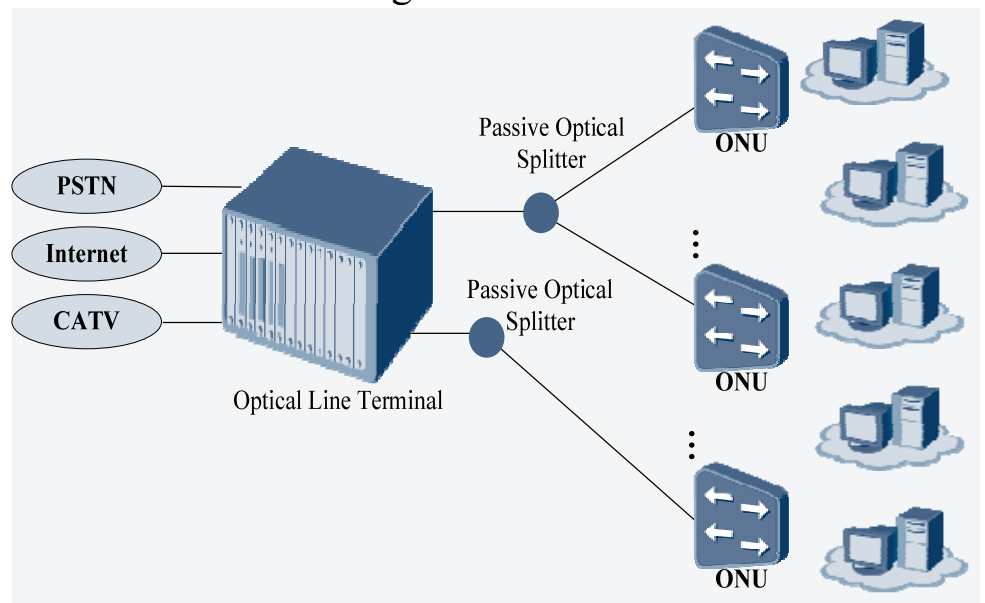

Figure 3. PON network 
In this network, OLT is a terminal device, it connects fiber trunks and provides varieties of services, which is like the switch or router in traditional communication network. ONU is a user side device, used to end the services from OLT. ONU works with OLT, can provide various services. ODN is a FTTH optical cable network that based on PON devices, which is used for providing optical transmission channel between OLT and ONU.

\subsubsection{EPON}

EPON (Ethernet Passive Optical Network) is a PON technology which is based on Ethernet. It is standardized by the IEEE802.3 EFM working group in June 2004, IEEE802.3 EFM working group released the EPON standard___IEEE802.3ah (incorporated into the IEEE802.3-2005 standard in 2005).

EPON is one of the new application technologies of optical access network. It can complete a single point to multipoint mode communication to realize the structure construction [4] and the transmission of data through the passive optical fiber mode. At the same time, it can provide integrated services to support a large number of functions that based on Ethernet platform.

\subsubsection{GPON}

GPON (Gigabit-Capable Passive Optical Network) is also a kind of passive optical network, and it is the latest generation of ITU- broadband passive optical access standard based on TG.984.x standard, with the advantages of high bandwidth (downlink rate close to 2.5Gbps), high efficiency, and large coverage. It provides the full service guarantee of QoS and service access while carrying ATM and GEM frame.

GPON is originated from the early APON $\backslash B P O N$ technology of optical fiber communication, so the transmission stream is used in the ATM and GEM frames. As for EPON, it is required to be able to seamlessly connect with the Internet, so the code is the Ethernet frame format. Of course, in order to adapt to the optical fiber transmission, EPON defines a layer of frame format out of the frame of Ethernet.

The PON network is introduced in the Section 4. We use both EPON and GPON in the PFTTH system.

\section{System Applications}

Yunnan Power Grid Corp is closely linked to the national Internet plus strategy and key work of China Southern Power Grid Corp. It constructs PFTTH experimental project in Yuxi, and then verifies the feasibility to develop communication services in the aspects of technology and engineering.

In this paper, we carry out the schemes of the arrangement of cable, measurement automation, intelligent power service, internet access and network security.

\subsection{Cable Layer}

The cable layer consists of $10 \mathrm{kV}$ cable distribution network and the part of PFTTH. There is a new-type 24-core ADSS (All Dielectric Self-Supporting) or common optical fiber cable, which length is about $4.2 \mathrm{~km}$, for the $10 \mathrm{kV}$ cable distribution network. The parts of PFTTH include feeder optical fiber cable, distribution optical fiber cable, household optical fiber cable and operator interconnection cable.

In the project of Yuxi, we deploy a set of 576-core optical fiber patch panel (MODF) for the welding, wiring, jumping of all optical fiber cables in the low-voltage distribution room, then 
deploy a set of photoelectric transfer box, for the storage, separation, wiring of the feeder cable OPLC in the area T3. As for area $\mathrm{T} 1$ and T2, in the strong current floor of $3 \mathrm{~F}, 6 \mathrm{~F}, 12 \mathrm{~F}, 18 \mathrm{~F}, 21 \mathrm{~F}$, $27 \mathrm{~F}, 33 \mathrm{~F}$ (for area $\mathrm{T} 3$, is $1 \mathrm{~F} 、 6 \mathrm{~F} 、 12 \mathrm{~F} 、 16 \mathrm{~F} 、 21 \mathrm{~F} 、 27 \mathrm{~F} 、 33 \mathrm{~F}$ ), we deploy a set of electric floor configuration cable separating box in each floor for housing, household wiring, as well as the indoor power network and outdoor power network ONU. In the area T3, photoelectric junction boxes replace the cable branch boxes which number are 1 in $1 \mathrm{~F}$ and 2 in $16 \mathrm{~F}$.

The overall cable topological graph is shown as Figure 4.

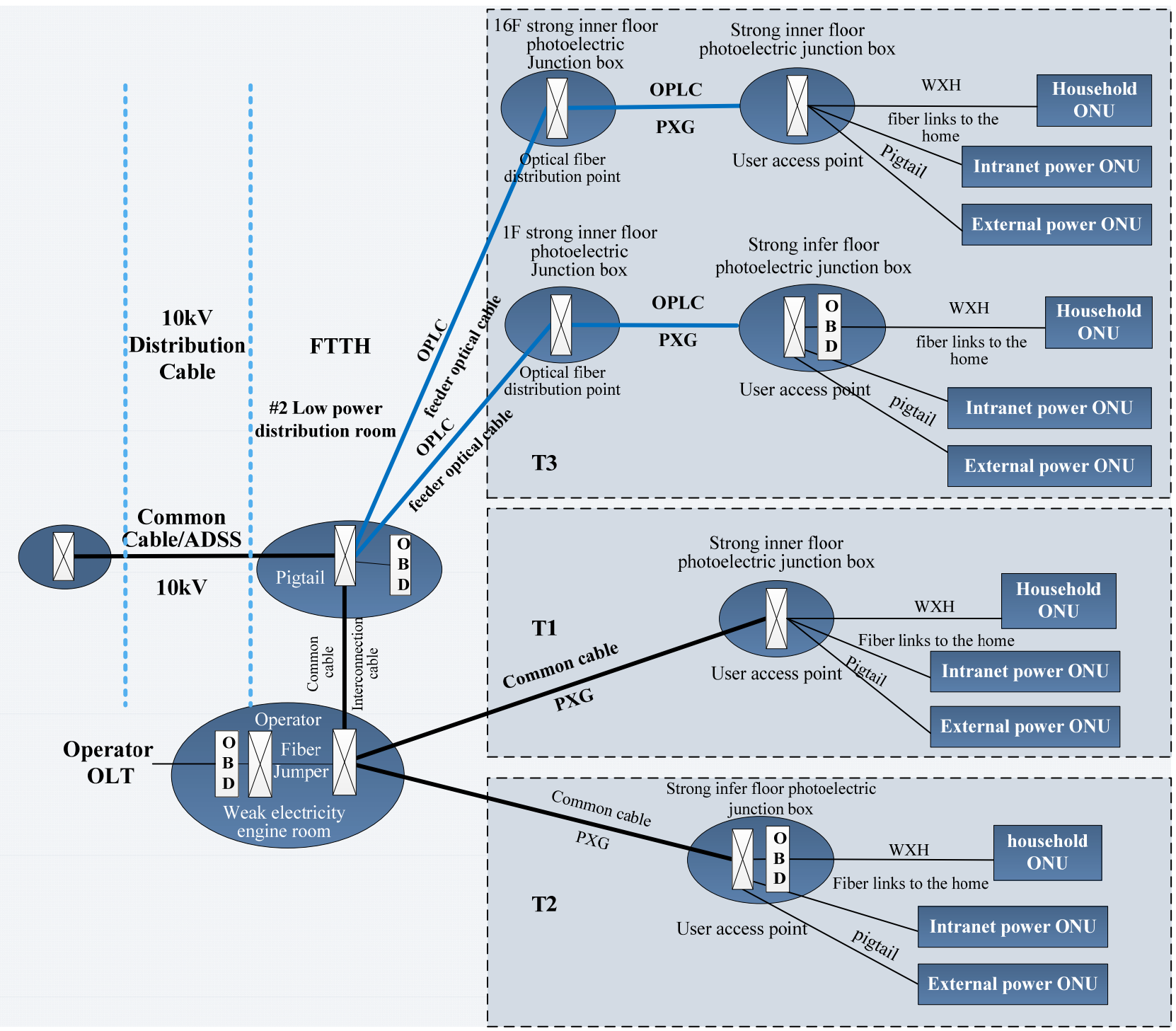

Figure 4. Overall cable layer topology

\subsection{Measurement Automation}

This system service uses the access point (AP) as in figure5, which is developed independently, to achieve user load, electric energy, voltage information collection, and realizes online monitoring of measurement device.

In this paper, the load management and low voltage concentration belong to the control area service; the electric energy data acquisition and processing belong to the uncontrolled area service; 
and web publishing belongs to the huge management information area service. The information collection frequency of the software is 1 time every 15 minutes, so the demand for communication speed is raised. The three-phase smart meter data is approximately $12 \mathrm{k}$ bytes, only collects data one time a day. It is obviously that the communication rate from concentrator to the local is not high.

The communication between measurement automation terminals gives priority to wireless public network, and is supplemented by other forms of communication. The terminals which are covered network, give preference to private network communication.

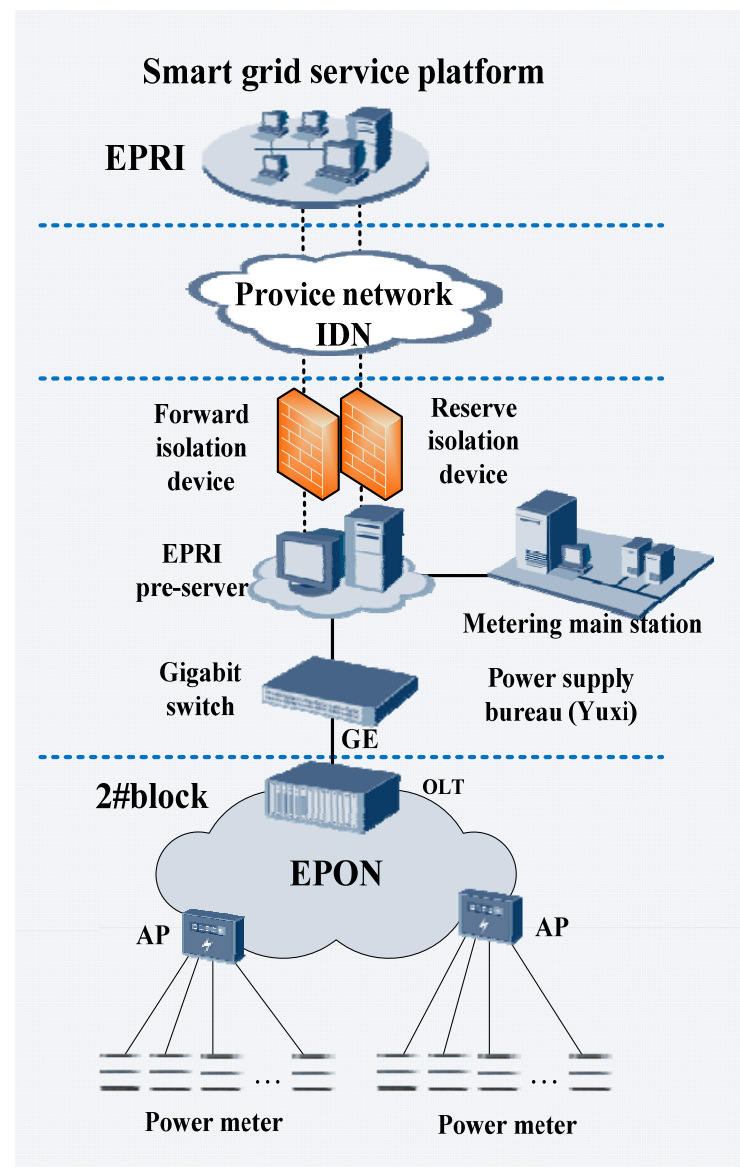

Figure 5. Measurement automation service access architecture

For Electric Production Recourse Agency (EPRA) business access security, as well as power network security protection, this project deployed two sets of positive and negative isolation device to ensure network service platform. These devices are procured by the science and technology project of EPRI, as shown in figure 5.

\subsection{Intelligent Power Service}

According to the characteristics of the experimental project construction, the intelligent power service is realized by the intelligent home system, which is based on wireless communication technology.

The electric and operation information of household appliances is transmitted through the socket 1, using Wi-Fi, to the indoor energy management terminals, and is further sent to the master server. Via the Internet, mobile phone, TV, laptop, tablet computer management terminals access grid service platform provided by the server (handheld power APP). The network operators control signals that transmitted to the indoor energy management terminal. To achieve remote control, 
indoor energy management terminal sends a signal through the socket, to the intelligent household appliances. At the same time, the information collected from the socket can be transmitted to the power grid service platform, so that the background energy consumption of the meter data can be analysed. Grid service platform requires docking with 95598 site data to get electricity fees, user power data and other user information.

The indoor energy management terminal connects with the Internet to achieve intelligent remote control and energy management of household appliances. At the same time, through the energy efficiency management platform, reasonable suggestions for the implementation of the ladder price can be given. Intelligent power network structure is shown in figure 6 .

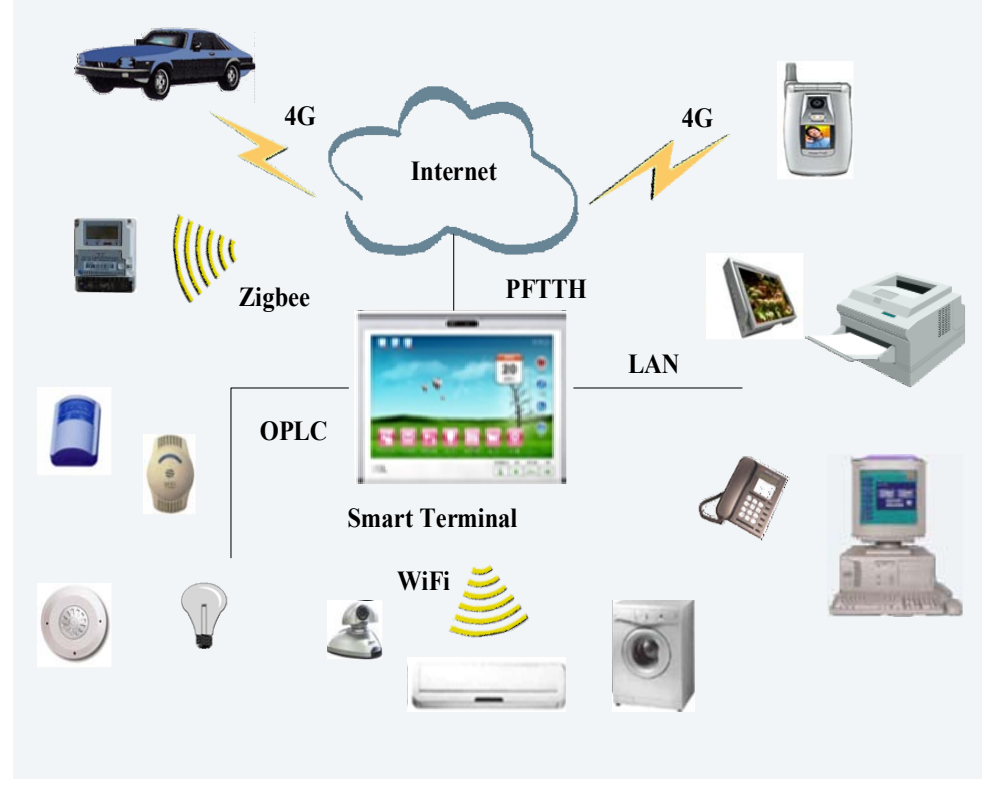

Figure 6 Structure of Smart power network

\subsection{Internet Access}

The network layer includes remote power distribution communication network and local communication network. 


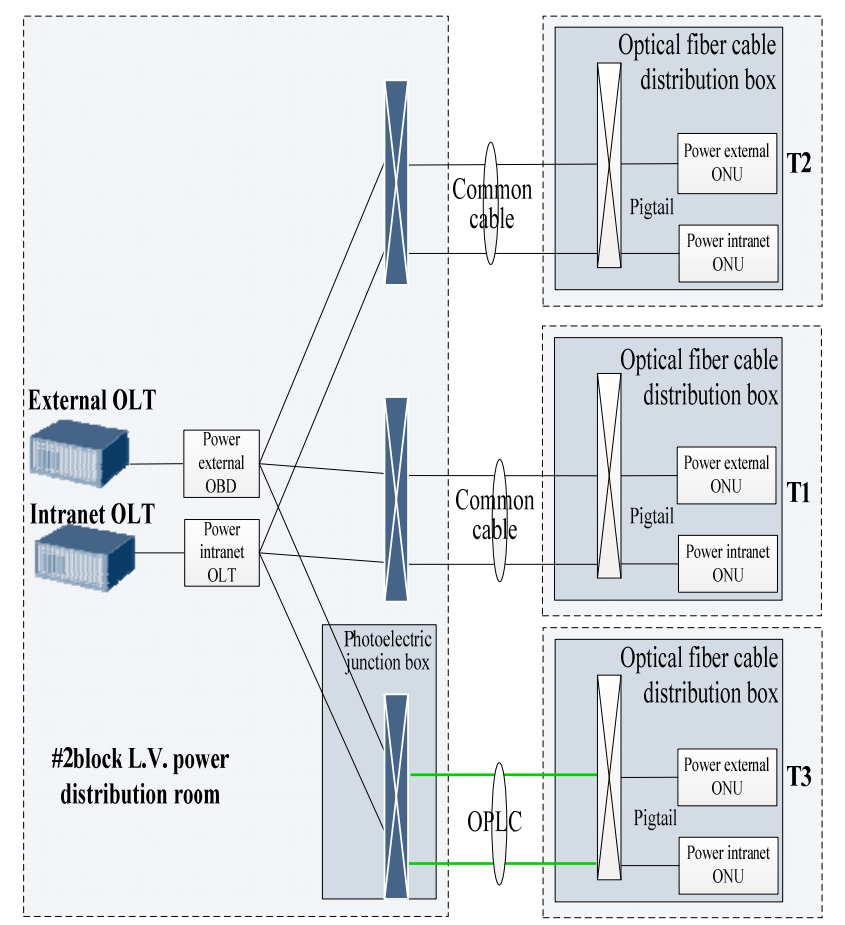

Figure 7 power local communication PON network

Remote communication network connects to China Unicom Internet export by deploying a network with three-layer switch, a firewall connection and a set of three-layer switch configuration. Through the metering station of the front server access area, the network carries services of the security of distribution automation, measurement automation and intelligent electricity [5][6]. Local communication network uses PON network scheme designed of double physical isolation in access network: configures two sets of OLT equipment in the distribution room. One set uses GPON technology for measurement automation service access, and the another set uses EPON technology for intelligent electric interactive information access. The power distribution local communication network of the power system is shown in figure 7.

As for the Internet access security protection, there is a set of Broadband Remote Access Server (BRAS) broadband remote access server, a set of authentication and billing server, and a set of firewall. The architecture is shown in figure 8. 


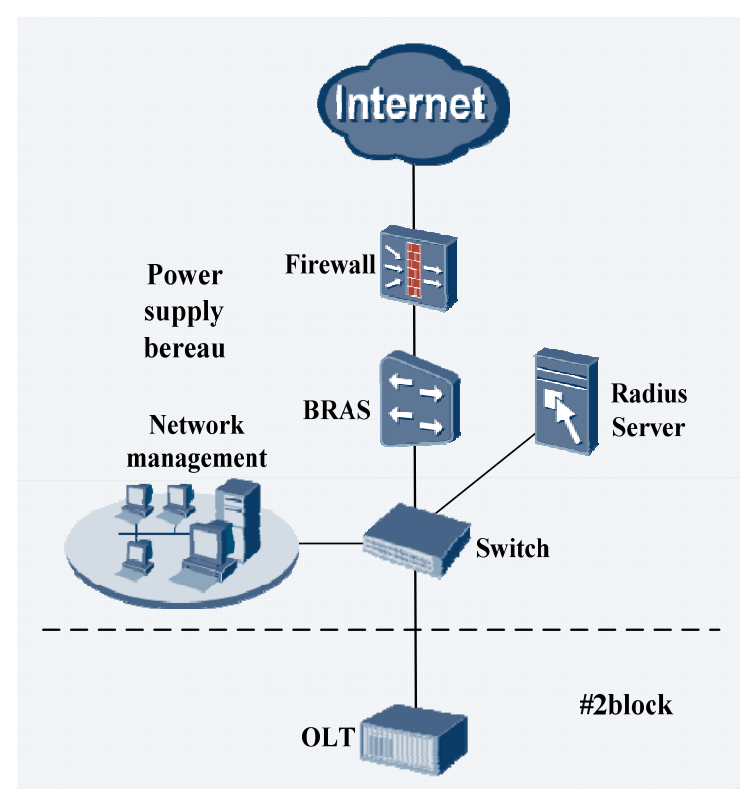

Figure 8 Security protection of Internet access

\section{Conclusion}

This paper is based on the PFTTH experimental project in Yunnan, Yuxi. We elaborate the key technologies and the actual system structures. The introduction of OPLC technology and the study of PFTTH xPON network technology are detailed, and the PFTTH system network architecture is proposed. Based on OPLC and xPON (including EPON and GPON) access network technology, PFTTH system can greatly promote the realization of intelligent use of electricity, and the construction costs can be reduced. This paper introduce several typical services of the PFTTH system in Yuxi. Accelerating the construction of intelligent electricity district can reap the optimal social and economic benefits, which is pivotal to achieve the most reasonable application of the smart grid resources.

\section{REFERENCES}

[1] Shuyong Chen, Shufang Song, Lanxin Li, et al. Survey on Smart Grid Technology, Power System Technology Vol.33, pp. 1-7, Apr 2009(In Chinese).

[2] Molin, Denis, P. Sansonetti, and P. Sillard. "Hybrid single and multimode optical fiber for a home network." EP, EP2657734. 2013.

[3] Liu Jianming1, Zhao Bingzhen2, Zhuang Zichao, The smart grid multiutility services platform based on power fiber to the home, in: Proc. of IEEE CCIS'11, 2011, pp. 17-22.L.

[4] Kakuma, Satoshi, et al. "Point-to-multipoint connection system.", US5487063. 1996.

[5] Yao Keng, GUO Tianwei, IIN Wen-long, HAN Bao-jia. Reduce the Loss by Using Single-Phase Power Distribution Technology[C]. Jiangsu Province Institute of Electrical Engineering Urban Electricity Supply Symposium, 2007.

[6] Zhang Hongqi, Li Fenghe, Bai Chengchun. Analysis of Energy Saving Efct of Single-phase Distribution Technology in Distribution Network[I]. SHANX ELECTRICPOWER,200S,36(2):53-55. 